\title{
TÉLÉVISION LOCALE ET PROXIMITÉ
}

\author{
Pierre Fastrez $^{1}$ et Stéphanie Meyer ${ }^{2}$
}

\section{Introduction}

Cette analyse de télévisions locales s'inscrit dans le prolongement d'une réflexion plus large de Caroline Huynen à propos de l'importance prise par la "proximité" à notre époque ${ }^{3}$.

Importance dans nos sociétés postmodernes d'abord, où différentes transformations d'ordre social semblent engendrer un repli de l'individu sur le proche, repli décrit en termes d'excès d'individualité $^{4}$, de nouvelles formes d'intimités, ou encore d'agrégation tribale ${ }^{6}$. Un transfert émotionnel profond s'opère : plus personne ne croit en l'espace public, et l'espace privé, désormais seul centre d'intérêt des

1 Aspirant au Fonds National de la Recherche Scientifique (F.N.R.S.).

2 Assistante au Département de communication de l'Université catholique de Louvain.

3 C. HuYnen, "La proximité dans l'esprit du temps", Recherches en communication, $\mathrm{n}^{\circ}$ 7, 1997, pp. 181-198.

4 M. Augé, Le sens des autres, Paris, Éd. du Seuil, 1994.

5 B. CAthelat, "Repli frileux", Autrement, n' 81, juin 1986; D. Madelenat, L'intimisme, Paris, P.U.F., Littératures modernes, 1989.

6 M. MAfFesol, "Une forme d'agrégation tribale", Autrement, $\mathrm{n}^{\circ} 98$, avril 1988 ; M. MAFFESOLI, Le temps des tribus. Le déclin de l'individualisme dans les sociétés de masse, Paris, Méridiens Klincksiek, 1988.

Recherches en communication, $\mathrm{n}^{\circ} 11,(1999)$. 
individus est l'objet d'un surinvestissement ${ }^{1}$. Face aux désillusions et à une société qui assaille les individus d'informations en tous genres, "l'homme postmoderne semble replier les ailes de ses songes sur l'espace intime d'une quotidienneté-refuge: foyer, famille, voisinage"2.

Importance dans les sciences humaines aussi, ce repli sur le proche poussant les chercheurs à effectuer un retour sur le sujet, là où l'ère structuraliste s'était évertuée à dégager des règles immanentes et des systèmes. "Avec la mise en valeur du sujet, c'est l'étude de la vie quotidienne, de 1' «ordinaire du sens» (...) qui acquiert sa pertinence"3, synthétisent Armand et Michèle Mattelart.

Importance dans les médias enfin, qui, en tant qu'acteurs sociaux, suivent ou engendrent les évolutions de nos sociétés et participent aux phénomènes évoqués ici. Corrélativement à l'avènement du cocooning et des nouvelles formes d'intimité, le passage de la paléo- à la néo-télévision, née de l'explosion de l'offre de programmes ${ }^{4}$, a fait du média télévisuel une présence amicale constante, disponible en permanence, avec laquelle on entretient une relation de complicité et de connivence ${ }^{5}$. La télévision est aussi devenue actrice : elle intervient désormais dans les débats de société. Au travers des reality shows, valorisant l'expérience privée et le témoignage profane, elle établit avec son public un pacte compassionnel ${ }^{6}$ reposant sur une double logique : "exhibition de l'individu, de sa souffrance"7 d'une part, et "identification [du public] aux malheureux et (...) empathie avec les souffrants"8 de l'autre, rompant par là le caractère purement interindividuel de la compassion, et rendant cette dernière partageable. Dans ce cadre, les limites des espaces public et privé se brouillent.

1 G. Lipovetsky, L'ère du vide. Essais sur l'individualisme contemporain, Paris, Gallimard, coll. Les Essais CCXXV, 1983.

2 D. MADELENAT, op. cit., p. 11.

3 A. et M. MATtelart, Penser les médias, Paris, La Découverte, 1986, p. 98.

4 Le développement de chaînes concurrentes a entraîné un accroissement et une diversification de l'offre des programmes, de sorte que tout type d'émission est désormais disponible chaque jour sur des chaînes différentes: "La télévision est passée d'une proposition de vente au détail de produits spécifiques à un hypermarché où l'on trouve de tout" (A. NEGRI, P. SiGNORELLI, R. DE BERTI, "Scènes de la vie quotidienne", Réseaux, $\mathrm{n}^{\circ} 44 / 45,1990$, p. 59).

5 Ibid., pp. 55-83.

6 D. MEHL, La télévision de l'intimité, Paris, Éd. du Seuil, coll. Essai politique, 1996.

7 lbid., p. 212.

8 Ibid. 
Télévision et médias suscitent de ce point de vue des avis contrastés. Certains perçoivent nos sociétés médiatiques comme génératrices d'aliénation, opérant une "spectacularisation" de nos rapports à autrui". Ainsi Richard Sennett affirme-t-il: "les médias ont énormément accru la quantité des connaissances que les groupes sociaux ont les uns des autres, mais ils ont également rendu tout contact réel superflu"2. D'autres, comme Maffesoli, y voient une occasion de vibrer (virtuellement) en commun, de communier avec la globalité sociale. A ce titre, la télévision locale constituerait une occasion de démassification des programmes télévisés et de leur spécification visà-vis de publics restreints, offrant une possibilité d'affirmer une identité culturelle propre ${ }^{3}$.

La recherche présentée dans cet article est ancrée dans ce contexte : s'interrogeant sur la notion même de proximité, et sur le rôle joué par les médias locaux dans les mouvements que nous venons d'évoquer brièvement, elle s'attache à examiner ce que signifie l'ancrage local d'une chaîne de télévision. Le terme "local" peut bien sûr être pris comme la limitation géographique très claire du territoire sur lequel émettent ces chaînes. Mais très vite, cette définition doit être élargie aux implications de cette limitation : le ciblage d'un public localisé, la sélection des informations en fonction d'un territoire restreint, créant peut-être un sentiment d'appartenance, et s'inscrivant dans une logique de société où il est de plus en plus question -nous l'avons vu- de "proximité". Nos questions peuvent être résumées comme suit : y a-t-il une façon de faire de la télévision locale ? Si oui, en quoi celle-ci peut-elle ou non être qualifiée de télévision "de proximité".

Les télévisions communautaires et locales de Belgique sont des médias relativement jeunes ${ }^{4}$ dont la diffusion est limitée géographiquement. Nous nous centrerons sur deux télévisions voisines mais apparues à des moments historiques différents, ne disposant pas des mêmes ressources et adoptant des philosophies de programmation différentes.

1 M. AugE, Non-lieux. Introduction à une anthropologie de la surmodernité, Paris, Éd. du Seuil, $1992 ;$ M. AUGÉ, Le sens des autres, op. cit.

2 R. SENNETT, Les tyrannies de l'intimité, Paris, Éd. du Seuil, 1979, p. 220.

3 J.-P. LAFRANCE, "Les télévision locales en Europe", Communication, n 51, 1990, pp. 221-242.

4 Les premiers projets ont été sélectionnés en 1976. 
Basée à Ottignies (à $30 \mathrm{~km}$ de Bruxelles), TV-Com est chargée de couvrir la province du Brabant wallon. Elle fait partie des premiers projets de télévisions locales et communautaires retenus en 1976 mais qui ont pourtant attendu plusieurs années pour pouvoir diffuser. Pendant la période analysée, TV-Com diffusait trente minutes de programmes par jour, constitués essentiellement d'un journal télévisé quotidien et de magazines d'information thématiques (sport, emploi, patrimoine, culture, etc.) ${ }^{1}$.

A Bruxelles, les projets de télévisions locales francophones se sont longtemps heurtés à la nécessité d'obtenir la signature du ministre de la Culture flamand, vu le statut bicommunautaire de la capitale. Un obstacle que Télé Bruxelles surmontera fin 1984. Aujourd'hui, elle émet en français sur les dix-neuf communes de la Région bruxelloise par voie de câble. Elle tente aussi de s'adresser aux francophones qui habitent dans les communes flamandes de la périphérie par voie hertzienne mais le résultat est peu concluant. Diffusés sept jours sur sept, ses programmes sont essentiellement des émissions de culture et d'information (journaux quotidiens, émissions d'actualité, etc.) $)^{2}$.

Depuis la fin du stade "expérimental" en 1985, les télévisions locales et communautaires sont des structures privées dont les missions relèvent du secteur public ${ }^{3}$. Elles doivent avoir une programmation dirigée sur l'information locale, le développement culturel et l'éducation permanente, et s'engager à respecter une zone de diffusion bien définie.

Avant d'exposer les résultats d'une analyse fouillée d'une semaine de programmation des deux chaînes retenues, nous présentons ci-dessous un modèle théorique centré sur la notion de proximité, qui servira de base à notre analyse, visant à répondre aux questions posées plus haut.

1 En dehors des heures de diffusion des programmes, la chaîne (re)diffuse en boucle les trente minutes de production quotidienne (la nuit) et un programme de vidéotexte (l'après-midi).

2 Télé Bruxelles propose également un vidéotexte ainsi qu'un télétexte.

3 Leur fonctionnement est régulé par le décret du 17 juillet 1987 modifié par celui du 19 juillet 1991. 


\section{Un modèle de la proximité médiatique}

En toute généralité, la proximité peut être définie comme étant le propre d'une relation réunissant deux sujets (ou objets), et impliquant par là même un minimum de différenciation entre ceux-ci, ce qui la distingue de la fusion pure et simple. Intrinsèquement dynamique, elle oscille constamment entre deux pôles opposés, pôles dont la nature est variable (proche - loin, familier - étranger, imminent - lointain, etc. ${ }^{1}$ ). La notion de proximité comporte en son sein une dimension appréciative qui la rend dépendante du contexte socioculturel dans lequel elle apparaît (ce qui est proche dans une culture ne l'est pas dans une autre $)^{2}$.

Si la proximité peut être définie par rapport à une relation impliquant deux sujets, dans toute communication médiatisée, on se trouve face à une situation plus complexe, car la situation comporte nécessairement non plus deux mais trois interactants : le destinataire (spectateur-auditeur-lecteur), le médiateur ${ }^{3}$ (télévision-radio-journalordinateur), et le monde référentiel ${ }^{4}$. Il convient donc de distinguer trois types de relation de proximité ${ }^{5}$ celle qui unit destinataire et médiateur, destinataire et monde référentiel, médiateur et monde référentiel.

1 De nombreux auteurs ont tenté d'établir des typologies de proximité fondées sur de telles distinctions. Cette question ne sera pas abordée ici. A ce sujet, cf. notamment: G. AuClair, Le Mana quotidien, Anthropos, 1970; Ph. BACHMANN, Communiquer avec la presse écrite et audiovisuelle. Paris, Les guides du centre de formation et de perfectionnement des journalistes, 1994 ; Cl. FURET, Le titre. Pour donner envie de lire, Paris, CFJP, 1995 ; J.-L. MARTIN-LAGARDETTE, Les secrets de l'écriture journalistique : informer, convaincre, Paris, Syros, 1987.

2 Cette définition s'inspire très largement de l'article de C. Huynen cité plus haut.

3 Par médiateur, nous entendons aussi bien le média-outil technique, que son personnel et sa culture d'entreprise, sa philosophie et ligne de programmation, etc.

4 Soit la part du monde réel dont le média traite dans ses programmes. On peut aussi parler de "monde raconté".

5 La proximité est le propre d'une relation, et non la relation elle-même. Par facilité, nous parlerons cependant de «relation de proximité» pour désigner cette caractéristique spécifique d'une relation donnée. 


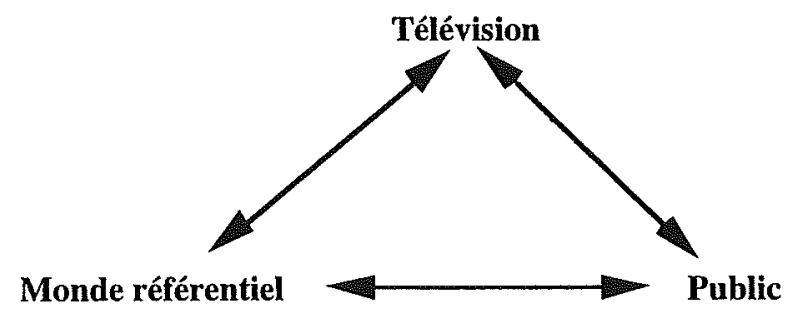

Pour chacune de ces trois relations, nous opérerons deux distinctions : la première distingue deux dimensions au sein de la proximité ; la seconde discerne deux types, deux états de proximité.

Dimension objective, dimension subjective. Toute relation de proximité impliquant deux sujets comporte selon nous deux dimensions : d'une part une dimension objective, qui réfère à une réalité tangible, souvent factuelle, toujours rationnellement évaluable ${ }^{1}$ (on est objectivement proche ou éloigné), et d'autre part une dimension subjective, correspondant à des données non rationnelles, tenant à la subjectivité (et particulièrement à ses sentiments, ses affects) de l'un des deux sujets impliqués dans la relation (on se sent subjectivement proche).

Cette distinction appelle deux remarques. Tout d'abord, et conformément au caractère relatif de la notion de proximité, la première dimension n'est "objective" que dans le cadre restreint d'un contexte socioculturel donné, au sein duquel les différents membres d'une communauté partagent des appréciations communes quant à ce qui est proche et ce qui est lointain. Ensuite, la dimension subjective d'une relation de proximité donnée peut différer selon le sujet impliqué dans la relation sur lequel on se centre : deux sujets peuvent être objectivement proches, mais ne pas se sentir proches de la même façon. De même, le substrat "rationnellement évaluable" qui fonde la dimension objective de la relation de proximité peut lui aussi différer d'un sujet à l'autre (dans une même relation): ainsi en va-t-il dans le cas où l'un des sujets possède plus d'informations sur l'autre que ce dernier sur le premier, comme cela peut être le cas du public d'une télévision par rapport au monde référentiel de celle-ci.

1 Ce substrat "factuel" pouvant correspondre tant à une distance physique qu'à une appartenance de classe, une nationalité, voire le fait de posséder des informations sur l'autre, bref, à tout élément pouvant être évalué de façon objective -dans un contexte socioculturel donné- car n'étant pas le fait d'une subjectivité. 
Proximité inscrite, proximité induite. La proximité inscrite est celle qui caractérise la relation unissant les deux sujets "au départ", sans que ceux-ci n'interviennent pour la modifier. Elle peut être considérée comme un "en-soi" pour une situation de communication donnée. Par opposition, la proximité induite témoigne d'un travail dans le chef de l'un des deux sujets, ou d'une tierce instance, visant un rapprochement supplémentaire par rapport au stade de la proximité inscrite. On parlera désormais de proximisation, terme ayant l'avantage de connoter un certain travail, un processus d'ajout, un traitement.

Notons que la séparation des deux types de proximité est uniquement analytique : dans la pratique, qui dit relation (de proximité) unissant deux sujets, dit inévitablement interaction entre ceuxci, et donc modification constante des paramètres de cette relation par les interactants (pour autant que ceux-ci soient en présence l'un de l'autre, présence réelle ou médiatisée)... ce qui ne rend pas illégitime le fait de supposer un stade "inscrit" à la relation d'un point de vue analytique. Dans le cadre d'une analyse empirique, on ne peut par contre procéder que sur base d'indices de saturation : quand il y a renforcement, quand manifestement ce qui est inscrit dans les faits est redoublé, accentué par un traitement, alors nous pourrons affirmer que les deux types de proximité sont présents.

Croisons maintenant les deux distinctions exposées. Une relation de proximité comporte à son stade inscrit une dimension objective et une dimension subjective. De même, le travail de proximisation peut porter tant sur la dimension objective de la proximité que sur sa dimension subjective : je peux me rapprocher objectivement de quelqu'un, tout comme je peux faire en sorte que nous nous sentions plus proches. Dans le premier cas (la proximisation objective), le travail de proximisation porte donc sur un rapprochement (ou une mise à distance) effective des deux sujets 1 , sur base de critères "rationnellement évaluables". Dans le second cas (la proximisation subjective), ce travail se centre sur la mise en valeur de la proximité existante (dimensions objective et/ou subjective), en visant un accroissement de l'importance de la dimension subjective de la proximité dans le chef d'un ou des deux sujets de la relation, c'est-à-

1 ... ou d'un sujet par rapport à l'autre (si la proximité objective se fonde sur l'information détenue par les sujets). 
dire une plus grande implication affective du ou des sujet(s) en présence dans la relation.

\begin{tabular}{|l|l|l|}
\hline & proximité inscrite & proximité induite \\
\hline dimension objective & "je suis proche de..." & $\begin{array}{l}\text { rapprochement/mise à distance } \\
\text { effectif } \\
\text { = proximisation objective }\end{array}$ \\
\hline dimension subjective & "je me sens proche de..." & $\begin{array}{l}\text { mise en scène, mise en valeur } \\
\text { = proximisation subjective }\end{array}$ \\
\hline
\end{tabular}

Si la distinction entre proximisation objective et proximisation subjective semble utile au niveau théorique, elle est difficilement applicable pratiquement, tout travail de proximisation n'excluant que rarement totalement l'une des deux dimensions. Nous parlerons donc en termes de dominantes (proximisation à dominante objective $v s$ à dominante subjective).

Jusqu'ici, notre exposé nous fournit une base conceptuelle permettant de distinguer (au sein de la triade médiatique) trois relations de proximité, comportant chacune deux dimensions, ces dernières pouvant chacune faire l'objet d'un travail de proximisation. En outre, ce travail de proximisation peut être l'œuvre soit de l'un des deux sujets impliqués dans la relation, soit d'une tierce instance.

Dans un tel contexte théorique, notre question de départ peut donc être formulée comme suit : dans une triade médiatique dont les relations entre pôles sont caractérisées par un état de proximité inscrite donné, quels sont les procédés auxquels les chaînes de télévision locales recourent pour influer sur cette proximité inscrite, pour mener un travail de proximisation à travers ses programmes ?

Ceci implique de relever -dans les programmes analysés-d'une part, les indicateurs témoignant des relations de proximité inscrite qui unissent télévision, public et monde, et d'autre part (et surtout), les indicateurs d'un travail de proximisation opéré par le média télévisuel ${ }^{1}$ sur ces trois relations (Télévision-Public et Télévision-Monde en tant qu'instance prenant part à la relation, et Public-Monde en tant que tiers intervenant dans la relation).

Notre investigation empirique a été guidée par l'hypothèse suivante :

1 Nous avons aussi observé quand et pourquoi ce travail intervient en rapport à la proximité inscrite. 
Plus les relations de proximité inscrite qui unissent les trois instances d'une triade médiatique donnée (télévision, public et monde) sont fortes ${ }^{1}$, moins le média (i.e. la chaine de télévision) de cette triade aura à mener un travail de proximisation sur la dimension subjective de ces trois relations de proximité, ce qui ne l'empêchera pas de mener un travail de proximisation objective sur celles-ci.

En l'occurrence, une télévision locale devra moins jouer sur la proximisation subjective qu'une télévision nationale, étant donné la proximité inscrite qui caractérise les relations qu'elle entretient avec son public et avec son "monde de référence" et que ceux-ci entretiennent entre eux.

\section{Analyse des programmes de TV-Com et Télé Bruxelles²}

Des trois relations de la triade médiatique, c'est la relation Public-Télévision qui a fait l'objet des plus nombreux travaux.

\section{Relation public - télévision}

La télévision, comme tout média, a des propriétés spécifiques, dont certaines peuvent être envisagées en termes de proximité inscrite. Comparée à la presse hebdomadaire, quotidienne et à la radio, la télévision apparaît comme le média de l'immersion (du direct), de l'émotion, de l'affectif, de la non-distance réflexive.

Si cette dimension a toujours marqué la télévision, son mode de consommation a évolué. La plupart des familles possédant (au moins) un téléviseur, celui-ci a pris place au cœur du foyer. "Le spectacle n'est plus situé dans un lieu public spécialement affecté à cet usage, il entre dans l'espace de la vie privée"3, expliquent Missika et Wolton. La télévision, compagne omniprésente de notre quotidien, n'est plus le média des grandes occasions exceptionnelles, "il est possible que la télévision, plus qu'un appareil électroménager, soit un animal domestique avec lequel on joue, auquel on s'intéresse et dont il suffit de

1 C'est-à-dire plus la proximité inscrite entre ces instances est importante...

2 Soit la production des deux chaînes du 28 mars au 3 avril 1998.

3 J.-L. Missika, D. Wolton, La folle du logis, La télévision dans les sociétés démocratiques, Paris, Gallimard, 1983, p. 165. 
sentir la présence"1. De même, le pacte communicationnel émetteurrécepteur a évolué en conséquence, comme le synthétisent Negri, Signorelli et De Berti :

L'idée d'une télévision écoutée comme un parent bon et sage a disparu et (...) elle a été remplacée par celle de l'amie du cœur. Si le parent inspirait et supposait le respect, l'amie, elle, porte à la complicité et à la confidence. Le rapport entre émetteur et récepteur se présente alors comme un rapport paritaire, bidirectionnel, amical et de confiance ${ }^{2}$.

Ces différentes caractéristiques (immersion, quotidienneté, relation de complicité) de la télévision débouchent sur une proximité inscrite entre le média et le public.

En dehors des grandes caractéristiques du média télévisuel, les télévisions locales ont leurs propriétés spécifiques. N'étant diffusées que dans une zone limitée, elles ne concernent que les spectateurs qui habitent dans un espace géographique donné. Il existe donc une proximité géographique de facto entre le public, le média (situé dans la même localité), et le monde auquel les émissions font référence.

Le relevé au sein des programmes des implicites relatifs à la connaissance de la chaîne, et plus particulièrement des "intertextualités" (référence à un programme dans un autre) doit permettre d'évaluer quel est le degré de connaissance supposé du public sur sa télévision locale. Cependant, ces indices sont trop rares dans notre corpus pour conclure sur le stade inscrit de cette relation de proximité. Pour les deux chaînes, le rappel -par le présentateur de l'émission en cour de diffusion-du programme qui suit constitue la seule trace d'intertextualité.

Le travail de proximisation objective opéré par les deux télévisions est par contre différent. Télé Bruxelles recourt régulièrement à l'invitation à la participation active du téléspectateur: dans des émissions (Coupe ta télé, Art et buffet) ou des annonces de programmes (Quartiers en questions), le spectateur est invité à téléphoner pour gagner des places de cinéma ou pour participer à un jeu. La conclusion de l'émission Larsen lui demande même d'écrire pour donner son avis sur le programme et proposer des améliorations.

1 A. Negri, P. Signorelli, R. DE BerTi, op, cit., p. 59.

2 Ibid., p. 59. 
Nombreuses, ces adresses ne sont pourtant pas accompagnées d'information sur leurs effets.

La présence du public se manifeste par contre parfois de manière "déléguée". Si les micro-trottoirs et interviews de quidams sont rares, dans Larsen, la présentatrice interroge des gens dans la rue et leur fait présenter les nouveaux CDs de la semaine. On notera l'existence d'un public de supporters dans l'émission Quartiers en question.

Sur TV-Com, les traces de proximisation objective sont quasiment absentes (pas d'émissions impliquant la participation du public). Les bandes annonces et autres formes de discours autoréférentiel n'informent le public que sur les sujets et horaires de diffusion des émissions. Il n'y a donc pas de la part de la chaîne de volonté d'informer son public de ses activités.

Qu'en est-il de la proximisation à dominante subjective? Dans le paysage audiovisuel contemporain, la concurrence entre chaînes, "la liberté d'action accordée au public et le fait que le rapport avec le téléviseur devient quotidien obligent l'émetteur à garder de façon continue (et non plus épisodique) l'attention du spectateur"1, expliquent Negri, Signorelli et De Berti. Face à cet impératif et à leurs moyens limités, nos deux chaînes réagissent de façon similaire.

A défaut de pouvoir entretenir le "flot" constamment comme les chaînes nationales, TV-Com et Télé Bruxelles ont opté pour la diffusion en boucle de leurs programmes. La télévision bruxelloise ajoutant la rediffusion d'émissions du week-end pendant la semaine.

Ces solutions procurent aux chaînes une présence constante, sans pour autant maintenir réellement le contact. On retrouve ici la position "d'animal de compagnie fidèle" que prend de plus en plus la néotélévision ${ }^{2}$. Le mode de diffusion adopté par Télé Bruxelles propose cependant une grille horaire plus construite que celle de TV-Com, donnant au téléspectateur l'illusion de "s'adapter" à ses activités, chaque programme étant visible à plusieurs moments de la semaine.

Negri, Signorelli et De Berti mettent en exergue l'importance accordée -dans la néotélévision- au rôle du médiateur intermédiaire (présentateurs ou animateurs) qui personnifie la châne et garantit le rapport de confiance égalitaire entre le public et l'écran ${ }^{3}$. Sur ce point, Télé Bruxelles se mobilise plus que TV-Com: les fins de JT se

\footnotetext{
1 Ibidem, p. 61.

2 Cf. supra.

3 A. Negri, P. Signorelli, R. De Berti, op. cit.
} 
concluent classiquement par l'annonce de l'émission qui suit, non pas en l'appelant par son titre, mais en prévenant le spectateur qu'il va retrouver "Mélissa" et "Jo Gérard". Balises de la chaîne, ces animateurs présentent également de courtes annonces pendant la semaine pour signaler les prochaines rediffusions de leurs émissions. Ce procédé est absent sur TV-Com. Certes, chaque émission est représentée par un journaliste unique, mais celui-ci utilise toujours le "nous" (aux rares occasions où il parle de l'émetteur du message). Il n'existe pas de programme mené par une "figure de proue".

Examinons à présent le travail de proximisation subjective opéré par chaque chaîne à travers la gestion sémio-pragmatique de la relation avec son public. Ici encore, TV-Com se montre relativement discrète : aucune trace d'énonciation n'est relevée dans les discours tenus à l'antenne, à l'exception du "nous" qui mentionne l'action de la rédaction d'un sujet, et des formules d'usage pour introduire un reportage ${ }^{1}$.

Dans le corpus considéré, les marques d'énonciation ou d'interpellation du téléspectateur sont limitées aux formules d'ouverture et de clôture de l'émission ${ }^{2}$, en dehors desquelles, le style du présentateur reste purement descriptif, constatif. Mémo Week-end, l'agenda culturel de la chaîne, recourt par contre toujours à l'utilisation du "vous" pour qualifier le spectateur ${ }^{3}$. Ces marques d'adresse viennent compenser la distance induite par le dispositif télévisuel utilisé, basé sur une voix off (énonciateur non identifiable) commentant les renseignements pratiques figurant à l'écran.

Du point de vue du mode d'énonciation, en dépit de dispositifs reposant sur l'axe Y-Y, les journaux produits par TV-Com ont tendance à tenir plus du registre du discours que de celui du récit, même dans les segments d'émissions ou l'énonciateur est nécessairement on, visible. Dans les reportages, l'énonciateur est systématique-

1 Comme "Reportage : Jean Dupont" dans Espace Région ou comme "Jean Dupont était dans les gradins lors de cette rencontre" dans les magazines sportifs.

2 Soit "Madame, Monsieur, Bonsoir" et "Excellente fin de soirée" pour le JT, "Bonjour !" et "Au revoir, à la semaine prochaine" pour Itinéris, "Bonsoir à tous", et "C'est sur cette très bonne nouvelle que nous nous quittons ce soir. Excellente soirée" pour Espace Sport, et "Bonsoir à tous, très heureux de vous retrouver dans votre Gradins !" pour Gradins (magazine sportif).

3 Exemples : "Le centre culturel de Rixensart vous propose ..." ; l'évangile "qui vous sera commenté par..." ; etc. 
ment $o f f{ }^{1}$. Ses seules traces sont perceptibles dans les interviews lorsqu'il doit relancer l'interviewé (la première question n'est presque jamais audible ${ }^{2}$ ). En dehors du présentateur, les journalistes ne sont donc que des noms. Au sein d'un dispositif basé sur le face-caméra, qui en soi est l'archétype de l'interpellation, on peut s'étonner de cette sous-utilisation de paramètres gérant la relation au public, qui nous conforte cependant dans notre hypothèse.

Les caractéristiques énonciatives des programmes de TV-Com se retrouvent dans les journaux télévisés et émissions d'information de Télé Bruxelles : leurs présentateurs sont généralement très sobres et interpellent rarement le public ${ }^{3}$. La tendance s'inverse par contre dans les autres émissions, où marques d'énonciation et d'adresse se mêlent. La présentatrice de Coupe ta télé lance régulièrement des "Télé Bruxelles vous invite à la première de...", "Télé Bruxelles et moimême vous invitons à ...", tandis que le présentateur de D'art d'art annonce une émission reprenant "la culture comme vous l'aimez". A ces quelques phrases, il faut ajouter le langage familier et attitudes décontractées de ceux qui mènent les émissions. Dans l'émission Larsen, la présentatrice va jusqu'à lui faire signe de la suivre, la "réponse" étant suggérée par le mouvement de l'image. Les marques d'énonciation peuvent aussi prendre des formes plus particulières : lors du décès d'un animateur de la RTBF, ancien de la chaîne, le présentateur annoncera "A sa famille, nous adressons nos plus sincères condoléances". Télé Bruxelles prend donc part au deuil, s'inscrit dans la "vraie vie" par son attitude "humaine" face à un drame.

Si le travail de proximisation subjective opéré par Télé Bruxelles vis-à-vis de son public semble plus important que celui fourni par TVCom, le contraste s'accroît encore dans les séquences studios du

1 Pour synthétiser, le recours à la voix off implique une position d'énonciation supérieure, émanant d'un sujet englobant, qui prend la parole pour tout le monde, et une centration dans le chef du récepteur sur ce point de vue omniscient. En revanche, l'énonciation on permet d'incarner l'énonciateur dans un individu particulier, le statut de ce qui est énoncé se limitant au point de vue d'un seul individu. Ces affirmations sont bien entendu à nuancer en fonction des aspects linguistiques et vocaux qui positionnent le point de vue énoncé. Cf. J.-P. MEUNIER et D. PERAYA, Introduction aux théories de la communication, Bruxelles, DeboekWesmael, 1993, pp. 248 et suiv.

2 Une seule exception a été relevée sur le corpus.

3 Deux cas ont été relevés sur la semaine. Le premier est le fait du présentateur qui dit "Comme vous le savez", l'autre survient en plein reportage et annonce "Si vous avez des observations (...)". 
magazine 4/5 de l'Iceberg (produit par la Communauté française de Belgique, et diffusé sur toutes les TVLC francophones).

La gestion de la relation au spectateur est ici prise à bras le corps, assumant totalement l'énonciation on et misant abondamment sur les marques d'énonciation et d'adresse. Nous sommes dans le registre du discours, et non plus du récit. Le présentateur, jeune, habillé décontracté, joue le rôle de médiateur entre le public, les "pingouins" (quatre comédiens chargés d'introduire les reportages par une improvisation) et les reportages. Il s'adresse ainsi constamment au spectateur et aux comédiens, en utilisant soit le "vous"1, soit le "nous" inclusif $^{2}$ ou exclusif ${ }^{3}$. Il devance ainsi les attentes, les questions, les réactions du public, et se base sur celles-ci pour introduire chacun des sujets. A la fin de l'émission, il laisse ainsi le dernier mot au public avec un "A vous de juger..." Tout ceci manifeste l'intention de l'émetteur (plus distant que les télévisions locales) de rendre la relation entre télévision et public la plus conviviale possible. On peut également relever le recours au style oral de certains textes ${ }^{4}$, à l'humour et à une préférence pour les plans rapprochés, voire les gros plans (en fin d'émission) induisant une proximité subjective supplémentaire, vu leur effet dramatique.

Notre hypothèse semble donc se confirmer sur ce point : en termes de proximisation accomplie par chaque émetteur, 4/5 de l'iceberg devance Télé Bruxelles, qui surpasse TV-Com. La distance de l'émetteur à son public donne le résultat inverse: TV-Com se limite au Brabant wallon, Télé Bruxelles s'adresse au tout Bruxelles, et l'émetteur de $4 / 5$ de l'iceberg a pour public toute la Communauté française de Belgique. Le rapport entre proximisation (en particulier subjective) et proximité inscrite semble donc inverse, comme nous le postulions plus haut.

1 Au spectateur: "Je suis sûr que vous vous posez la question"; "Vous avez en mains quelques données qui vous permettent de mieux comprendre ..."; etc. Aux pingouins : "Etes-vous prêts ? Et bien c'est à vous, quand vous le voulez...", etc.

2 "C'est ce que nous allons voir tous ensemble tout au long de cette émission", etc.

3 "Aujourd'hui nous vous parlerons du travail dans le secteur de l'économie sociale", etc.

4 Ex. : "C'est quoi pour vous, le secteur de l'économie sociale?". 


\section{Public - Monde référentiel}

Toute télévision locale et communautaire doit couvrir une zone géographique donnée en termes d'informations diffusées, en vertu du décret de 1987. La sélection d'informations concernant cette zone relève donc de la proximité inscrite. Mais le monde référentiel des télévisions correspond-il à ces zones ?

Pour TV-Com, la réponse est oui : zone couverte et monde référentiel ont les limites de la province du Brabant wallon. Ainsi, sur une semaine de journaux télévisés, seuls deux des événements relatés dépassent les frontières de la province, les deux tiers des sujets étant du ressort d'une seule commune. Les événements d'ampleur régionale ou nationale ne sont abordés qu'en étant réduits à une dimension provinciale ou locale. De même, $86 \%$ des écrans du vidéotexte portent sur un événement à échelle communale, $9 \%$ sur un événement concernant deux communes, et $5 \%$ sur le Brabant wallon entier. Par contre, dans la publicité, seuls deux spots concernent un événement et une firme du Brabant wallon. Au journal télévisé, quatre sujets d'ampleur provinciale sur cinq sont annoncés dans les titres et font donc l'objet d'un reportage complet, ce qui marque l'importance accordée à la province.

Les choses sont moins claires sur Télé Bruxelles. Classés en fonction du niveau de l'information, les 55 sujets du Journal se répartissent en 4 catégories $^{1}$ : quinze au niveau communal, six entre les niveaux communal et bruxellois (régional), quinze de niveau bruxellois, sept entre les niveaux bruxellois et national, huit de niveau national et quatre de niveaux national et international. $65,5 \%$ des sujets sont donc d'un intérêt communal ou régional, ce qui est dans la logique de proximité inscrite des télévisions communautaires.

Plus surprenants sont les 19 sujets qui dépassent ce cadre. Dans notre catégorisation, le niveau national reprend les expositions (qui ne portent pas sur des thèmes locaux), les informations sur les communes à facilités, les informations sur l'organisation de "Bruxelles 2000 capitale culturelle". On retient également une série d'événements de portée générale mais dont les aspects les plus visibles se déroulent à Bruxelles (manifestations devant le siège de sociétés, défilés d'étu-

1 On suppose qu'un sujet de niveau général a des implications plus locales, l'inverseétant rarement le cas. 
diants, ouverture de Child Focus, lancement d'un système d'archivage audiovisuel à l'ULB, compétitions sportives au Heysel, etc.).

Ceci nous mène de la proximité inscrite à la proximisation à dominante objective : en relayant des événements de portée extrarégionale, Télé Bruxelles rapproche son public bruxellois du reste de la Belgique ou de l'Europe. Elle présente Bruxelles comme point de cristallisation, comme lieu important au regard des non Bruxellois. Ceci est confirmé par l'existence de l'émission Quinze consacrée à l'Europe dont Bruxelles est la capitale, mais aussi par une rapide comparaison entre le $18 \mathrm{~h}$ de Télé Bruxelles et le $19 \mathrm{~h} 30$ de la RTBF qui traitent de six sujets communs sur les quatre jours observés.

Le travail de proximisation objective mené par TV-Com sur son public vis-à-vis de "la jeune province" s'oriente selon deux tendances. D'une part, la chaîne diffuse une quantité importante d'informations pratiques permettant au public de participer aux événements de sa province, de sa commune, etc. D'autre part, une large part de l'information fournie sur le monde implique les institutions locales et provinciales.

Parmi les sujets des journaux télévisés, les événements ponctuels, à caractère exceptionnel ou festif, connotés positivement ${ }^{1}$ sont les plus fréquents (bien qu'ils ne fassent que rarement partie des titres). Les sujets portant sur l'aménagement des communes ou de la province, relativement nombreux, apparaissent -eux-fréquemment en titres. Outre ces sujets, on trouve encore, en dehors de titres, d'autres décisions prises au niveau communal: Les sujets portant sur la promotion de la province sont largement mis en avant : au seul de ces sujets qui n'est pas annoncé en titre, on consacre une interview plateau entière. Les institutions locales et provinciales sont donc bien en vue au sein des informations diffusées. Ainsi, les deux personnes interviewées dans le prolongement du journal télévisé (le mardi et le jeudi) sont un bourgmestre du Brabant wallon, et une attachée culturelle de la province.

Cette mise en avant des institutions locales et provinciales se double d'une possibilité de dialogue avec celles-ci offerte par la chaîne : le lendemain de l'interview du bourgmestre, le présentateur est amené à faire part au public d'un "droit de réponse" de personnes

1 Par opposition, par exemple, aux faits divers, événements ponctuels et locaux connotés négativement, qui sont absents, à une exception près. 
opposées à celui-ci dans un litige. La chaîne se pose ici comme médiateur entre les uns et les autres.

La volonté -déjà évoquée- de mettre des renseignements pratiques à disposition est prolongée par "Mémo Week-end", l'agenda culturel de la chaîne, détaillant cinq événements à venir (type, lieu, organisateur, téléphone). Le vidéotexte, diffusé en boucle l'aprèsmidi, prolonge cette démarche, avec un agenda culturel (20 écrans sur 44), un agenda sportif (15 sur 44), et de la publicité ( 8 sur 44$)^{1}$. Ces annonces sont donc des invitations à participer, susceptibles de rapprocher effectivement le public du monde : elles tiennent donc de la proximisation objective.

Sur Télé Bruxelles aussi, le domaine du politique apparaît comme largement dominant (11 sujets, plus 2 sur les institutions régionales et 2 sur la sécurité). Viennent ensuite les préoccupations urbanistiques : logements (4), nouvelles constructions (3) et espace public (5). Essentiellement de portée nationale, on relève également les conflits sociaux (5) et les problèmes dans l'enseignement (4). Enfin, les événements culturels représentent à eux seuls 6 sujets et le sport seulement 3. Comme sur TV-Com, la majorité des émissions culturelles de la chaîne mentionnent des détails pratiques concernant les événements renseignés.

Passons à présent à la proximisation subjective. Sur TV-Com, celle-ci s'opère essentiellement entre le public et les institutions, ce qui corrobore ce qui précède. C'est ce qui se passe quand la chaîne joue sur la mise en évidence de l'identité locale. Ainsi, dans le (traditionnel) faux reportage du journal du $1^{\mathrm{er}}$ avril, qui a pour thème le sentiment d'appartenance à la province du Brabant wallon ${ }^{2}$, la farce cite les autorités provinciales comme les instigateurs de l'événement, et est montée avec la complicité de celles-ci : une séquence montre le gouverneur commentant et fredonnant l'hymne fictif. L'appartenance à la province est donc considérée comme un caractère partagé par tous (chaîne, public, autorités), un lieu de connivence propice à faire de l'humour.

I Le vidéotexte peut aussi intégrer des informations d'intérêt général n'appartenant pas à ces rubriques. Sur la boucle recensée, un écran appartenait à cette catégorie : le déménagement d'un service d'une commune.

2 Afín de renforcer celui-ci, la députation permanente de la province aurait fait composer un hymne brabançon, que tous les écoliers des instituts provinciaux devraient entonner pour commencer la journée. 
De même, le magazine Itinéris, dédié au patrimoine brabançon, débute et se clôt sur une animation faisant apparaître le logotype du Brabant wallon, "la jeune province". Le même logotype orne un coin de l'écran durant les premières secondes de chaque reportage.

Les institutions provinciales sont encore présentes à travers le traitement de l'information au sein du journal télévisé. Deux tendances se dégagent. D'une part, l'angle de traitement de l'information est toujours localiste : si un sujet déborde du cadre provincial, son traitement s'arrête toujours à cette frontière. A titre d'exemple, voici comment la présentatrice d'Itinéris ouvre l'émission consacrée à la pisciculture :

Ce sont les Danois qui ont lancé la pisciculture à grande échelle. Aujourd'hui, on en trouve une trentaine en Région wallonne, et une seule en Brabant wallon. Elle est installée le long du Glabais, et coule des jours heureux et productifs.

En l'espace de deux phrases, le thème développé tout au long de l'émission a été réduit aux dimensions de la province. Pour les sujets de niveaux provinciaux ou communaux, l'angle d'attaque privilégie les conséquences dans une localité précise.

Mais le traitement n'en est pas pour autant humanisant, et fait souvent primer la fonction (institutionnelle) sur l'homme quand il recourt à la personnification de l'actualité. Sur la semaine considérée, aucun des reportages n'aborde son sujet à travers le cas d'un personnage particulier. De même, dans le cadre des reportages du JT, à deux exceptions près ${ }^{1}$, ne sont jamais interrogés que les "responsables" : conseillers communaux, agents de développement rural, délégués syndicaux, présidents d'intercommunales, etc. A chaque fois, la fonction prime sur l'homme. Même dans les manifestations, interviews de "Monsieur Tout-le-Monde" et micro-trottoir ne sont jamais utilisés.

Comme sur TV-Com, le traitement des sujets d'information est rarement humanisant sur Télé Bruxelles : un seul reportage porte sur l'initiative d'un particulier, on recense très peu de micro-trottoirs ou de séquences donnant la parole à des quidams. Télé Bruxelles ne semble donc pas faire du "local" stricto sensu, au sens des journaux quotidiens locaux. Dans cette même logique, on ne retrouve pas de

1 Deux participants à une journée porte ouverte, interrogés dans le même reportage. 
fait divers, à part la mention de fouilles suite à la disparition d'un enfant.

Aucune des deux chaînes n'exploite donc l'incarnation de l'actualité dans un personnage particulier, ce qui permet pourtant de corriger la "myopie" dont le public souffre face aux informations, concernant souvent de vastes ensembles humains par des implications à moyen ou long terme, et ce "en réduisant la distance psychologique qui sépare le public de l'information. Autrement dit en «sensibilisant» le lecteur et donc en introduisant des facteurs d'intérêt humain"'.

Une identité locale propre au Bruxellois peut aussi être dégagée des programmes de Télé Bruxelles, qui joue sur celle-ci. Ainsi, selon elle, le Bruxellois type s'intéresse à la politique régionale et, sans doute marqué par la "bruxellisation", soucieux des transformations de sa ville qui reste avant tout un lieu d'habitation. Il croise quotidiennement des défilés de mécontents venus de toutes les régions du pays, des supporters endiablés, etc. Côté sport, ce n'est pas la passion (peu d'informations sur ce sujet), au contraire de la culture. En plus des annonces d'expositions et spectacles dans les journaux télévisés, une panoplie d'émissions sont consacrées à ce domaine, de sorte que la culture est le second type d'émission après l'information ${ }^{2}$. L'analyse des annonces commerciales relevées du lundi au samedi précise ce portrait flou. Dans $89 \%$ des cas (65 sur 73), les publicités concernent des annonceurs locaux. Parmi elles, on constate une nette dominance $(24,5 \%)$ des annonces pour des manifestations culturelles et pour les loisirs, viennent ensuite l'habillement ( $22 \%)$ et l'aménagement de la maison $(15 \%)$, enfin on retrouve les transports $(7 \%)$, l'hôtellerie (7 \%), et d'autres thèmes $(13,5 \%)$. Le profil culturel du Bruxellois s'affirme donc et se double de l'image de quelqu'un qui prend soin de son apparence et de celle de son domicile.

Le magazine 4/5 de l'Iceberg (déjà mentionné) offre à nouveau une comparaison intéressante par rapport à TV-Com et TéléBruxelles. Ici, trois reportages sur quatre du magazine se focalisent sur les cas d'entreprises particulières, au sein desquelles les gens interrogés sont tant les patrons que les employés les moins qualifiés. L'un des reportages est d'ailleurs présenté comme un ensemble de témoignages du quotidien des personnes travaillant dans le secteur de

\footnotetext{
I A. KIENTZ, Pour analyser les médias : analyse de contenu, Tours, Mame, 1971.

2 Cf. annexe.
} 
l'économie sociale. On joue donc pleinement la carte de l'humanisation de l'information.

L'une des séquences de l'émission communautaire est produite par la chaîne de télévision qui la diffuse. L'annonce de cette séquence par le présentateur prend cette forme : "Alors, ça se passe comment, près de chez vous, l'économie sociale ?". Cette question présuppose que le public est à même de répondre, alors que le présentateur ne l'est pas. Celui-ci se pose donc comme distant du monde et du public pour mieux rapprocher ces derniers.

L'émission recourt chaque semaine à un micro-trottoir précédé d'un "Alors petite question, comme chaque semaine : c'est quoi, pour vous, le secteur de l'économie sociale ?". Les personnes interrogées (censées faire partie du monde de référence ${ }^{1}$ ) sont présentées comme une délégation du public. L'ensemble de ces procédés s'oriente vers un rapprochement subjectif du public et du monde de référence. Sachant que 4/5 de l'Iceberg est l'œuvre d'un émetteur distant, ceci confirme partiellement notre hypothèse.

\section{Relation Télévision - Monde référentiel}

Une chaîne de télévision peut ou non entretenir des liens de proximité (inscrite) avec les événements dont elle traite (cela se passe dans le même quartier, la chaîne a une appartenance politique marquée en faveur du parti donc elle parle...). Elle peut aussi chercher à se rapprocher de ce monde référentiel. Dans cette partie de notre recherche, nous avons été attentifs au travail de proximisation objective et à la façon dont les deux chaînes se présentaient à leur public comme proches de leur monde référentiel ${ }^{2}$. Les indicateurs pris en compte ont porté entre autres sur le discours autoréférentiel des chaînes, ainsi que sur les "lieux idéologiquement marqués"3 (citations d'autorité, interviews de spécialistes, etc. ${ }^{4}$ ).

1 La séquence en question a été tournée à Namur... qui n'appartient pas à la province du Brabant wallon.

2 On parlera de proximité mise en scène.

3 Nous faisons appel à l'indicateur de la grille de Jules Gritti (Cf. notamment G. RINGLET et alii, "Comment lire les journaux", in La presse quotidienne. Dossiers du CIMCS, Bruxelles, 1979 (dossier n³)).

4 La question nous guidant étant "Qui interroge-t-on pour s'enquérir d'un avis pertinent?". 
Deux types d'indices de proximisation à dominante objective ont pu être dégagés de notre corpus. Les premiers sont propres à un travail de proximisation mené au sein même des programmes de la chaîne. Sur Télé Bruxelles, celui-ci prend corps dans le fameux "débat sur notre antenne" du dimanche regroupant tout le gouvernement bruxellois "dans les locaux de Télé Bruxelles". Cet événement vaudra un reportage en troisième position dans le JT du lundi. La télévision s'y montre comme lieu exceptionnel de dialogue sur la crise entre ministres, membres de l'opposition et journalistes régionaux. Elle fait donc partie de l'actualité bruxelloise, voire la crée en partie. Le pendant d'une telle démarche sur TV-Com correspond aux interviews bi-hebdomadaires de personnalités, en page "invité" du journal télévisé. La diffusion du droit de réponse du $1^{\text {er }}$ avril -mentionnée plus haut-procède du même mouvement. Notons que sur TV-Com comme sur Télé Bruxelles, la proximisation porte sur le monde institutionnel de par le choix des invités.

Le deuxième type d'indices est relatif à un travail mené en dehors des programmes des chaînes, et repris dans ceux-ci en tant que proximité mise en scène. Ainsi le journal télévisé de Télé Bruxelles annonce-t-il qu'une convention pour créer un réseau européen de neuf télévisions locales a été signée et que "le bureau de coordination se situera dans les locaux de TV Bruxelles". Aucun indice de ce type n'a pu être relevé chez TV-Com.

La proximité mise en scène par Télé Bruxelles prend encore d'autres formes. On la retrouve notamment dans le générique de son journal : une fenêtre (l'emblème de la châ̂ne) se découpe dans un plan de Bruxelles, vu à travers un filtre bleu; les titres s'inscrivent ensuite successivement sous la fenêtre, accompagnés d'un commentaire. La caméra plonge alors à travers la fenêtre, et survole la partie sud-est de la Région bruxelloise (à dominante francophone ${ }^{1}$ ). Elle rentre ensuite dans le pentagone (le centre) et longe un tunnel bordé d'images d'actualité. S'affiche alors la phrase "Le Journal". Ce générique présente très clairement Télé Bruxelles comme une fenêtre sur la Bruxelles francophone et particulièrement sur le centre de la capitale. Ce survol terminant par un plongeon dans les images donne une impression de distance et donc de recul nécessaire pour pouvoir

1 Les communes flamandes seront plutôt couvertes par TV Brussel, chaîne régionale flamande. 
aborder pleinement ensuite les événements. Cette conception de "l'œil qui voit tout" est très classique.

L'inscription de la châ̂ne dans son monde référentiel se retrouve dans l'annonce déjà mentionnée du décès de l'animateur de la RTBF lorsque la télévision locale rappelle que c'est elle qui lui avait permis de faire ses débuts.

Les personnes interrogées dans les journaux télévisés nous permettent de préciser la part du monde référentiel dont chaque chaîne se présente comme proche. Télé Bruxelles recourt peu aux microtrottoirs ${ }^{1}$ et la grande majorité des interviewés sont des hommes politiques (15 sur 44 ) et autres hommes de pouvoir (6 présidents de sociétés, d'associations semi-publiques, etc.). Viennent ensuite des représentants de manifestations de protestations (6) et des comités de quartier (3). La parole n'est jamais donnée à des contestataires isolés, mais bien à des groupements reconnus, voire institutionnalisés. Les organisateurs de manifestations culturelles et artistiques ou sportives (5), ainsi que les spécialistes (5) forment la queue du peloton.

La tendance semble identique dans les programmes de TV-Com, où la fonction de l'interrogé prime toujours sur l'individu ${ }^{2}$. De même, la farce du premier avril induit une connivence entre autorités et chaîne. L'impression qui en ressort est celle d'une proximité, d'une connivence entre le journaliste et les "experts" du sport.

\section{Une hypothèse confirmée ?}

Plusieurs tendances émergent de l'analyse. Elles ne confirment qu'en partie notre hypothèse de travail. Commençons par TV-Com. Si le travail de proximisation subjective opéré par la chaîne (évoluant dans une triade médiatique où la proximité inscrite est élevée) est globalement faible, comme annoncé, les différences les plus marquantes se situent non pas entre proximisation objective et subjective mais vis-à-vis des différentes relations de la triade médiatique. La relation télévision-public est ainsi dédaignée par la chaîne (des points de vue objectif et subjectif), au profit de la relation monde-public (marques de proximisation subjective). Il n'en va pas de même pour

1 On n'en relève que, dans un sujet, l'interview d'une dame venue faire tatouer sa voiture, et, dans un autre, les réactions d'enfants participants à une opération pédagogique.

2 Cf. supra. 
Télé Bruxelles : les participation et présence de public délégué côté objectif, et les nombreuses adresses et autoréférences côté subjectif, opèrent une proximisation entre télévision et public. Mais ceci concerne essentiellement les programmes culturels, les autres informations étant présentées avec beaucoup plus de détachement. Si Télé Bruxelles semble ainsi se "couler dans le moule" des télévisions nationales marquées par le modèle de la néotélévision, c'est sans doute parce qu'elle a tendance à devenir régionale et non plus locale. S'éloignant ainsi de son public, elle serait obligée de compenser cette relative distance par un travail de proximisation.

Cet élargissement se marque aussi dans la relation public-monde référentiel : diffusant sur une région entière, la télévision s'adresse à des personnes de tous horizons. Dès lors, parler d'une fête de quartier inconnue pour ceux qui habitent à moins d'un kilomètre semble être une stratégie difficile. D'où l'intérêt pour des éléments qui concernent le plus grand nombre (politique régionale), qui sont visibles de tous (les manifestations), ou qui n'ont pas de réelle identité locale (événements culturels et artistiques).

Par sa sélection de sujets, Télé Bruxelles construit une image du spectateur bruxellois qu'elle présente comme homme de culture et attentif à son environnement, à sa ville et à son confort de vie. Une image tout à fait compatible avec celle qu'elle donne du monde bruxellois présenté comme lieu important pour ceux qui n'y habitent pas, et avec celle qu'elle donne indirectement d'elle-même comme média ambitieux à vocation plutôt régionale que locale. Ce jeu de proximisation subjective entre le monde et le public, viendrait donc compenser une information qui tend à s'éloigner des événements micro-locaux. Dans cette logique, notre hypothèse serait donc vérifiée en partie.

Une autre tendance de fond-observée pour nos deux télévisions- porte sur l'importance accordée au monde institutionnel. Ainsi, la proximisation opérée sur le public vis-à-vis du monde lui fait la part belle. De même, la proximité mise en scène entre télévision et monde institutionnel se voit accorder une importance de premier plan. Sans doute peut-on assimiler cette dernière tendance à une recherche de légitimité dans le chef de la chaîne, qui maintiendrait alors volontairement la distance vis-à-vis de son public afin de fonder celle-ci à l'ombre des institutions locales et (plus encore) provinciales pour TVCom, régionales pour Télé Bruxelles. A nouveau, la différence d'échelle entre TV-Com et Télé Bruxelles apparaît. Elle justifie selon 
nous (sur base de notre hypothèse de travail) le surcroît de proximisation subjective à laquelle Télé Bruxelles recourt par rapport à TVCom.

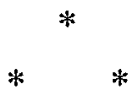

Nous voudrions pour conclure soulever une limite potentielle de notre méthodologie : la plupart des indices opérationalisant les concepts de notre cadre théorique (en particulier ceux portant sur la proximisation subjective, clé de voûte de notre hypothèse ${ }^{1}$ ) sont normalement conçus pour évaluer les effets de messages audiovisuels, et non pour débusquer les intentions de l'émetteur. Notre travail ayant pour but de dégager le travail de proximisation dans le chef des chaînes de télévision étudiées, il existe un risque de décalage entre nos observations et la nature de nos conclusions. C'est donc la question de la validité interne de ces dernières que nous posons $\mathrm{ici}^{2}$. Cependant, ayant résolu de centrer notre approche sur les productions des chaînes considérées, nous ne pouvions éviter ce biais car nous avons tenté de diversifier au maximum les types d'indices utilisés. L'inscription d'une telle étude dans une démarche plus large, englobant des approches complémentaires en production et en réception devrait palier ce décalage potentiel.

$\mathrm{Au}$ travers des conclusions énoncées ci-dessus, on peut malgré tout voir comment la notion de proximité (et ses subdivisions conceptuelles) permet d'appréhender le champ des télévisions locales, et de caractériser comment celles-ci se positionnent au sein de leur environnement (systématisé dans une "triade médiatique"). Les travaux de proximisation menés par une chaîne de télévision ne sont en effet pas fortuits, mais révélateurs de la façon dont la télévision se conçoit en tant qu'acteur de la société au sein de laquelle elle évolue.

1 Comme par exemple les marques sémio-pragmatiques d'énonciation du message audiovisuel.

2 Celle de la validité externe étant réglée d'avance : nos conclusions ne valent que pour les deux cas étudiés dans le cadre de cette recherche. 


\section{Annexe : liste des émissions diffusées par TV-Com et Télé Bruxelles}

\section{TV-Com}

titre

-Espace Région

-Espace Sport

-Itinéris

-Mémo Week-end

-4/5 de l'iceberg

$-B W$ Hebdo

-Gradins

\section{Télé Bruxelles}

titre
-Le Journal
-Coupe ta télé
-Conte à rebours
-4/5 de l'iceberg
-Larsen
-Extra-large
-Court toujours
-Quartiers en questions
-D'art d'art
-Art et buffet
-Ainsi Va la Ville
-Quinze

diffusion
quotidien
lundi
mercredi
jeudi

vendredi

samedi

dimanche

\section{nature}

joumal télévisé (15 min.); actualités sportives (15 min.) ;

magazine du patrimoine brabançon (15 min.) ;

agenda de manifestations culturelles diverses (5 min.) ;

magazine de l'éducation, la formation et l'emploi, produit par la Communauté française de Belgique (15 min.);

magazine d'actualités composé de 5 reportages diffusés dans Espace Région la même semaine ( $15 \mathrm{~min}$.) ;

actualités sportives (30 min.).

\begin{tabular}{|c|c|}
\hline diffusion & nature \\
\hline quotidien & journal télévisé ; \\
\hline quotidien & agenda culturel ; \\
\hline quotidien & petite histoire ; \\
\hline hebdomadaire $(*)$ & $\begin{array}{l}\text { magazine de l'éducation, la formation } \\
\text { et l'emploi, produit par la } \\
\text { Communauté française de Belgique. }\end{array}$ \\
\hline hebdomadaire (*) & magazine musical et culturel ; \\
\hline hebdomadaire $(*)$ & émission consacrée au cinéma ; \\
\hline hebdomadaire (*) & présentation de courts métrages ; \\
\hline hebdomadaire $(*)$ & jeu de culture générale pour les jeunes ; \\
\hline hebdomadaire $(* *)$ & émission culturelle ; \\
\hline hebdomadaire $(* *)$ & magazine de culture et restauration ; \\
\hline hebdomadaire $(* *)$ & magazine d'actualité ; \\
\hline bdomadaire $(* *)$ & émission consacrée à l'Europe ; \\
\hline
\end{tabular}

rem. : tous les programmes hebdomadaires sont rediffusés plusieurs fois en semaine.

(*) lère diffusion le samedi.

$(* *) 1^{\text {ère }}$ diffusion le dimanche. 